\title{
A multi-agent system for managing adverse weather situations on the road network
}

\author{
I. Martía , V.R. Tomás ${ }^{a, *}$, L.A. García ${ }^{a}$ and J.J. Martínez ${ }^{b}$ \\ ${ }^{\mathrm{a}}$ Ingeniería y ciencia de los computadores, Universidad Jaume I, Castellón, Spain \\ ${ }^{\mathrm{b}}$ Instituto de robótica, Universidad de Valencia,Valencia, Spain
}

\begin{abstract}
The development of traffic management and control strategies to improve traffic flows and road safety is necessary due to the high dynamism of traffic flows. The use of distributed intelligent systems can help the traffic organizations and the road operators to cope with possible incidents on the road network, especially when the incidents are related to adverse meteorological conditions. In that case, the probability of road accidents is increased due to the difficulty of driving under bad weather conditions. So, if the operators detect any meteorological incident, they must decide how to deal with it in order to improve traffic safety. In this paper we introduce a new multiagent system (MAS) to support traffic management when there appear meteorological problems in the road network. MAS technology helps to deal with the specific characteristics of traffic domain. The proposed MAS is able to work in two ways: a) coordinately, where all the agents work to solve weather problems in large networks and b) locally, where due to communications breakdown small groups of agents work together to inform road users about weather problems. The MAS has a rule-based system to deal with the meteorological data and decide the actions to take in front of any meteorological issue. This expert system also controls the quality of the data, improving the road operator confidence in the decisions taken by the expert system. However, weather sensors can provide wrong data, due to several factors (hardware failure, climate factors, etc.) so the rule based system controls these provided data by applying specific coherence and correlation rules to improve the quality of the taken decisions.
\end{abstract}

Keywords: Advanced traffic management systems, multiagent system, expert system, road incidents traffic management

\section{Introduction}

The application of modern information technologies to traffic management has made possible to apply advanced theories and knowledge models to enhance more sophisticated traffic control and management systems, the so-called Intelligent Traffic Systems (ITS). Advanced Traffic Management Systems (ATMS) are ITS whose purpose is to help traffic road managers on their ordinary traffic tasks by means of Decision Support Systems (DSS) [18].

The hard quest for traffic road managers is to improve traffic safety and mobility as much as possible. But, this task is very difficult to accomplish due to several factors:

* Corresponding author: Dr. V.R. Tomas, Ingenier_a y ciencia de los computadores, Universidad Jaume I, Castelln, Spain. E-mail: vtomas@uji.es.
- Road traffic operators have to manage huge road networks, involving several main and secondary roads. In normal conditions, the management of traffic is focused on main roads, as they have capacity to absorb more vehicles. However, in case of severe traffic problems, secondary roads could be used to redirect traffic flows.

- There is a great amount of equipment for road traffic monitoring. Furthermore, these elements are located in great distances one from each other and, generally, are laid in main roads (thus, traffic status estimation on secondary roads is a hard complicated task). Moreover, it is difficult the integration of these sensor data, not only due to the big amount of data these sensors provide, but also because the different time of data integration and the different measures provided by these traffic sensors (i.e. traffic density, traffic flow, etc.). 
- Traffic information is distributed between the different Traffic Control Centers (TCC) of the road network. This implies that if a traffic problem spans to several TCC, any TCC only has a partial vision of this traffic problem. So, there are needed coordination and negotiation protocols between several TCCs in order to effectively deal with the traffic problem.

- There are several organizations with competence, not only in traffic management, but also on other crucial tasks for traffic problem solving (road maintenance, civil protection, firemen, etc.)

In the last decades, advances in Artificial Intelligence have been applied for developing several ATMS to deal with some of the difficulties exposed (estimation of traffic capacity $[3,12,13]$; optimal sensor location [16], automatic incident detection using wavelets $[1,2,26]$; estimation of trip distribution models [5]; advanced car-following models [14]; traffic prediction models by qualitative simulation [20]; incident detection by using qualitative temporal simulation [7]; traffic light control using neural networks [19]; etc.).

Moreover, meteorological factors (rain, snow, ice, etc.) can severely impact traffic behavior by deeply decreasing safety and mobility [6]. Thus, due to the intrinsic distributed nature of the control, management and equipment of the traffic domain, the traffic actions to be performed to deal with traffic and meteorological problems may involve coordinate, cooperative and even also independent techniques to be applied.

From a software architecture and functionality perspective, a modern ATMS dealing together with several of the previously related factors naturally falls in a software agent architecture framework. In 1990, Ritchie introduced an ATMS to support the TCC operators of Intelligent Vehicle-Roadway System (IVRS) [25]. This system is based on multiple real-time knowledgebased expert systems (KBES) integrated by a distributed blackboard problem-solving architecture. In this architecture, the manager had to validate every report produced by the system. Hernández et al. described in 1999 the TYRS architecture [11]. TRYS is a knowledge representation environment, based on multiagent technologies, supporting models to perform traffic management at a strategic level in urban, interurban or mixed areas. In the TRYS system each agent is responsible of a concrete coverage area. The agents cooperate to support decisions about traffic management in urban and suburban networks. The power of TRYS lays in the implementation of dynamic traffic management measures that, analyzed by the global system, determine the mea- sures for the complete road network. Besides, it can be modified with new strategies, but the changes need to be made by computer engineers advised by traffic experts.

In 2003, the Track-R system was introduced. This system consists of a group of agents, responsible for a geographical area. These agents have to communicate between them in order to provide traffic routes recommendations for humans or other agents [8]. It is a closed system, i.e., it can not be modified to introduce new routes.

Another ATMS based on multiagent technologies was introduced in [27]. This system is based on traffic management plans and uses traffic ontology to define the road traffic domain. It includes a framework for negotiation between different TCCs, maybe belonging to different traffic administrations, in order to deal with incidents that affect more than a single coverage area.

All cited ATMS can support daily traffic management but the decisions on the traffic actions to execute are performed by a single agent, usually a manager agent which stores a general overview of the traffic status. However, if there is a communication breakdown between the TCC and the other elements of the traffic system, there is not an opportunity for isolated components of the traffic system to run up-to-date actions to deal with local problems (usually, these isolated traffic systems, in the presence of a communication breakdown, use to run pre-fixed control actions).

However, the use of local rule based systems $[9,17]$ could be applied to deal with the up-to-date local decision taken in the presence of the two possible scenarios (with and without active communications).

In order to solve these problems, it is exposed a new ATMS based on multiagent and rule-based system technologies to manage efficiently meteorological incidents. The ATMS is designed to work in two ways: local and coordinate. In the coordinate way (with active communications) all the agents work together to improve road safety in the presence of detected meteorological problems in large networks. In the local way (without active communications) agents work autonomously with respect to the local traffic information available.

\section{The traffic management system}

Different equipment is distributed along the road network, to monitor traffic information, analyze it and warn the users in case of meteorological issues. The relevant components for traffic management in adverse meteorological situations can be classified in [4]: 


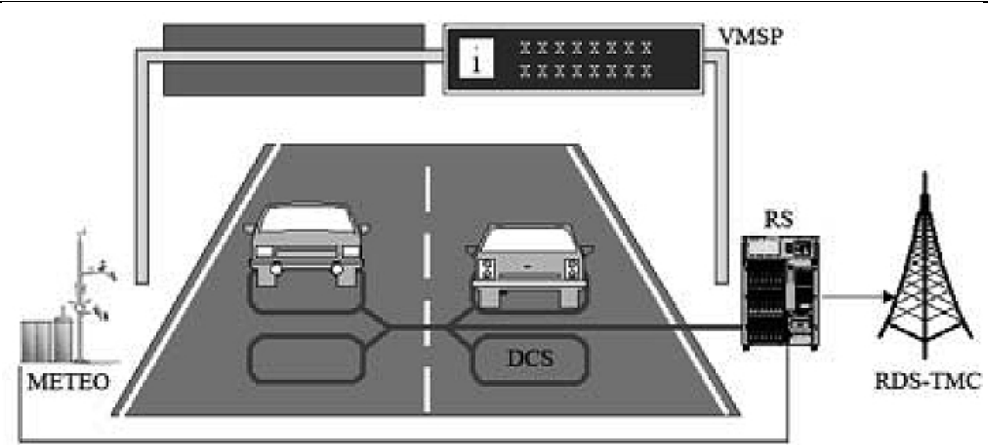

Fig. 1. Example of a local system. In this example, the local system has the RS with RDS-TMC module, a weather station, one VMS and four DCS.

- Road Traffic Monitoring

* Meteorological Station (MS), which collects data provided by meteorological sensors.

* Data Collection Station (DCS), which collects data about traffic on the road network.

- Information Systems

* Variable Message Signal (VMS), which shows messages to the users of the road network.

* RDS-TMC Module, which is a technology for delivering traffic and travel information to drivers via radio signals.

- Management

* Remote Station (RS), which controls MS and VMSs. The RS can also have a RDS-TMC module to inform the users via radio.

The designed traffic management system contains a TCC with several local systems. If communications between local systems and the TCC are available, the local systems will inform the TCC about meteorological issues on their controlled area. In that case, the TCC will take a decision to warn the users about potential problems resulting from the incident and will communicate the information to the local systems; whish will use the appropriate equipment to show that information to the road users.

The local systems can work independently of the TCC, so the equipment of the local system must be able to receive meteorological data, analyze it and warn the users in case of meteorological issues. This feature will be useful in case of breakdown in the communications between local systems and the TCC. This way, the system will be always able to alert users about weather problems.
The minimum equipment a local system must have, and the requirements of this equipment must meet, in order to work autonomously are:

- Local systems have at least one MS and one RS.

- The system can have any number of VMS. In case it has no VMS, the RS must have a RDS-TMC module.

- If the RS does not have a RDS-TMC module, the system must have at least one VMS.

- The system can have any number of DCS.

- The RS controls all the equipment in the local system.

Any possible configuration of a local system following these requirements allows the capture and monitoring of meteorological variables, their analysis to detect meteorological incidents and to inform the users, either by VMS or RDS-TMC.

Figure 1 shows an example of local system architecture. In this system, there is a RS with a meteorological station providing weather data. In order to control traffic flows, the local system has two DCS, one on each direction of the road. The local system has two ways to inform the users of the road network: via the RDS-TMC module or via the VMS that has on each direction of the road.

\section{MAS architecture}

Based on the features and equipment of the traffic management system presented in the previous section, an ATMS was designed to receive meteorological data from the road network, analyze it and warn road users in case of meteorological issues. This ATMS works with a heterogeneous set of agents. There are agents to deal with data capture and those who make an advanced 
4 L. Mart et al. I A multi-agent system for managing adverse weather situations on the road network

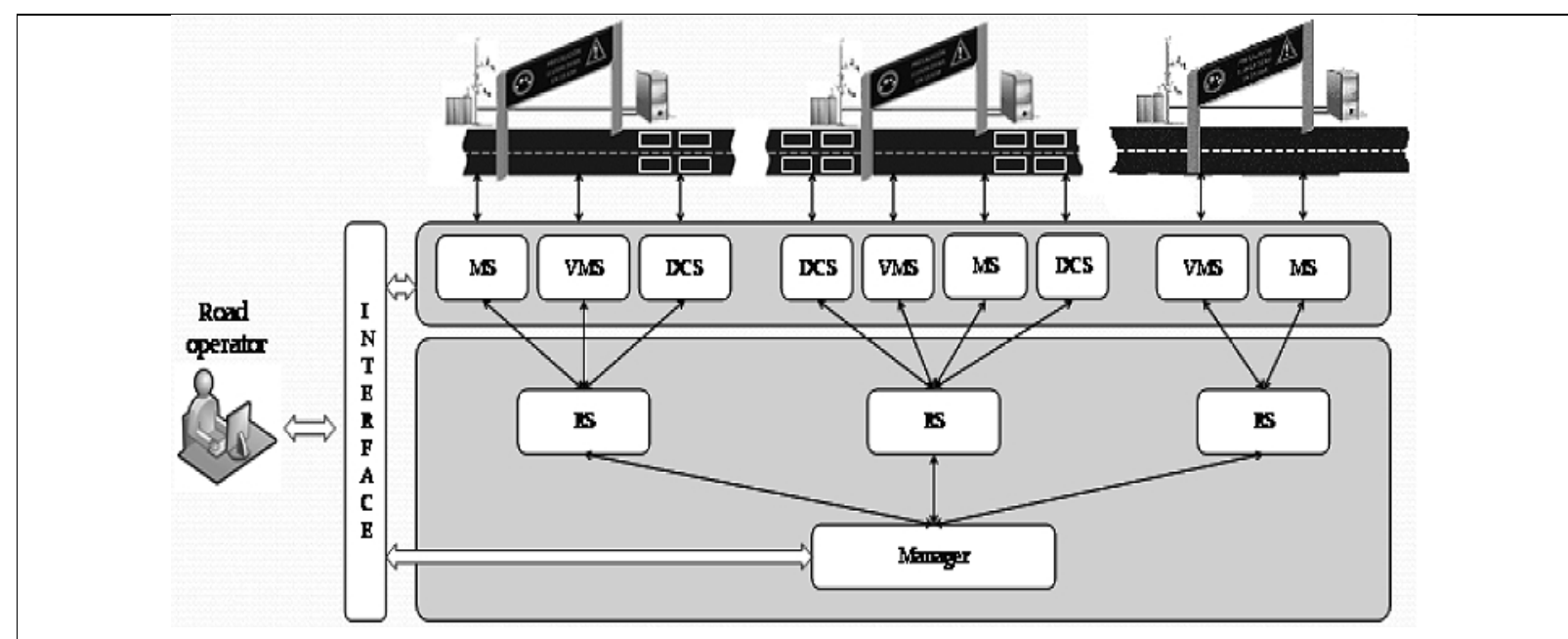

Fig. 2. MAS architecture. The image shows several local systems which are connected to the Manager agent via the RS agent. The road operator operates with the MAS through the interface. The VMS, DCS and MS agents are the ones that interact with the environment while the others perform the control actions.

analysis of these weather data and propose a decision to deal with the detected problems on the road network. The agents are defined based on the functionality of the equipment to which they are associated.

The Manager agent is set at the TCC and controls the behavior of the proposed MAS. Each RS has a RS agent associated, in order to control meteorological issues in local areas; each meteorological station has a MS agent; every DCS has a DCS agent; the VMSs have VMS agents; and the Interface agent will show the road managers the actions taken to avoid traffic problems

Figure 2 shows the architecture of the proposed MAS. The VMS, DCS and MS agents interact with the environment while the RS agent and Manager agent perform the management of the system. The road operator can interact with the complete MAS through the Manager agent. The MS, VMS and DCS agents send their information to the interface, so the road operator can see the state of the equipment.

A more concrete description of the functionality of each agent is described below.

\subsection{MS agent}

Each MS has an associated MS agent. This agent collects meteorological data provided by the sensors of the station, packs it and sends it to the RS agent. Moreover, it is able to recognize communication problems with the RS and sensor failure.

\subsection{DCS agent}

Each DCS of the road network has an associated DCS agent. This agent collects data about the traffic on the road network, packs it and sends it to the RS agent. Moreover, it is able to recognize communication problems with the RS and sensor failure.

\subsection{RS agent}

Every RS has an associated RS agent. This agent collects data from MS agent and analyzes it. If a meteorological problem is detected, it sends a notice to the Manager agent and an alert message to the most appropriate VMS for alerting users. If there are any problems in the communications with the TCC, the local system is able to work autonomously

The RS agent has a rule-based system whose knowledge base that contains the following information:

- Information about the road network. Describes and identifies the segments and links of the road network associated to the RS.

- Information about the elements of the local system (the meteorological station, the number of VMS, etc.).

- Meteorological issues. There are three types of rules to analyze meteorological data and to set the certainty of a meteorological issue.

* Detection rules, which are used to detect meteorological issues based on the meteorological data. These rules compare the value provided by 
L. Mart et al. I A multi-agent system for managing adverse weather situations on the road network

Priority matrix relating $\%$ of certainty (columns) with the risk factor (lines)

\begin{tabular}{lccccc}
\hline & $0-64 \%$ & $65-74 \%$ & $75-84 \%$ & $85-94 \%$ & $95-100 \%$ \\
\hline $91-100$ & 0 & 10 & 6 & 4 & 1 \\
$81-90$ & 0 & 14 & 8 & 5 & 2 \\
$71-80$ & 0 & 16 & 11 & 7 & 3 \\
$61-70$ & 0 & 19 & 15 & 12 & 9 \\
$51-60$ & 0 & 0 & 18 & 17 & 13 \\
$0-50$ & 0 & 0 & 0 & 0 & 0 \\
\hline
\end{tabular}

the sensors with a threshold in order to establish if there is any meteorological issue (rain, snow, etc.).

* Coherence rules, which check whether the provided sensor values are sound. These rules compare these values with historical values on the same dates, in order to check if these values are sound.

* Correlation rules, which are used to detect correspondences between data provided by several sensors to evaluate the quality of these data. These rules depend on the climate of the region where the RS is located. For example, in an area with a high relative humidity the temperature of the air uses to be higher than in one with a low relative humidity.

- Rules to control the VMS. There are some rules to decide in which of its VMS the RS agent must show the messages.

The fact base of the rule-based system contains the traffic and meteorological state in real time. This state is obtained by using the data sent by the MS agent and the DCS agent.

The inference engine of the rule-based system applies the rules of the knowledge base to the data of the fact base in order to decide if there are any meteorological problems and, in that case what is the priority of each problem. First, detection rules are applied to the meteorological data in order to find any issue. If a meteorological problem is detected, an alarm about that problem is fired, with a default degree of certainty (initially set at $85 \%$ ).

Once the alarm is set, the coherence rules check if the sensor values are sound. These rules check if the historical values stored in the RS are similar to the current values. In order to do this, the coherence rules compare these current values with previous values in similar dates and hours. If the current value is sound, the certainty of the alarm will be increased by $10 \%$. Otherwise, the certainty will be decreased by $10 \%$.

Finally, the correlation rules are applied. These rules have two functionalities:
1. Check data reliability. Data from a sensor is compared with data coming from other sensors to check if these values are coherent. For instance, it can't be snowing with $24^{\circ} \mathrm{C}$. If data from different sensors are coherent, the certainty is increased by $10 \%$; otherwise it is decreased by $10 \%$.

2 . Find non-existent data. If one sensor fails obtaining the data, information coming from the correlation rules can be used to estimate the value of this sensor. In that case the certainty is decreased by $10 \%$.

To set the priority of an alarm a priority matrix based on the certainty factor and the risk factor is used. The main purpose of this matrix is to highlight a certain situation involving a potentially high risk rather than uncertain situations involving high risk or certain situations with low risk. Thus this matrix sets a priority based on the balance between certainty and risk. The risk factor is an empirical value obtained from accident analysis in relation with meteorological conditions, traffic conditions and road characteristics [28].

Table 1 shows the priority matrix used to set the priority of the alarms. When the certainty of a meteorological phenomenon is lower than $64 \%$ or the risk factor of that phenomenon is lower than 50 , that phenomenon cannot cause problems in the road network, so it is not necessary to fire an alarm. In other case, it is better to report something that is high certain, rather than to report something that is low certain; but if something is not known to be $100 \%$ sure, but the appearance of this phenomenon can decrease the safety of the road an alarm has to be fired too. This way, a meteorological alarm has the highest priority when has a certainty higher than $94 \%$ and a risk factor higher than 91 .

In case of meteorological alarms, the remote station has to inform the TCC. To this end, the RS agent sends a message to the Manager with the meteorological issue and applies the protocol established by the Manager agent.

The RS is able to recognize a failure in the communications with the TCC. In that case the RS agent should 
take the appropriate actions to inform road users in the most appropriated way, that is, it has to warn users via VMS or RDS-TMC. If the RS controls more than one VMS, it has to decide which VMS is the most appropriate to warn users and send the meteorological alarm to it. If there is no VMS, the message should be sent using the RDS-TMC module.

\subsection{VMS agent}

The VMS agent is associated to the VMSs. Its mission is to control the messages shown on the VMS screen. When the VMS agent receives a message, it has to decide whether to show it at the VMS or not. In order to make that decision, the agent has a ruled based system which decides to put the message in the VMSs based on the priority of the message.

The knowledge base of the rule-based system of the VMS agent contains:

- Information about the VMS. Code of the road where it is located, kilometer, direction, number of lines, number of characters per line, total number of symbols and a variable to identify whether it is active or not.

- Types of messages. Messages are classified in four groups, from lower to higher priority

* Spreading: these messages spread information to the users like "Remember to use the seat belt".

* Information: the purpose of these messages is to prevent the user from a potential impact on the road such as congestion, construction, an accident, etc.

* Regulation: these messages show signaling mandatory for all road users, like max speed, required the use of snow chains, etc.

* Forced: such information can be of different meaning but it has been determined by an operator.

- Priorities of the messages. Different messages of the same type can have different priorities, set by the RS, based on the importance the message. For example, an information message of snow will be more important than information about rain, but both can be regulation messages about max speed.

- Rules to show the message. The rules to decide whether to show a message or not.

The fact base of the rule-based system contains the new message to show in the VMS. Also, if a message has been removed to put one with a higher priority, the removed message has to be stored at the fact base.

The inference engine of the rule-based system is based on the priorities of the. When a new message arrives at the VMS agent, if the VMS does not have any message, the new message is signposted on the VMS. If there is a message on the VMS, the inference engine has to decide if the new message has a higher priority than the current one. In that case, the message on the VMS will be replaced by the new one, the deleted message will be stored on the fact base and the RS agent will be alerted of the change. Otherwise, the new message will be stored on the fact base and the RS agent will be alerted of the inability to signpost the new message.

\subsection{Manager agent}

Every TCC is managed by a Manager agent. This agent decides which actions should be taken in case of meteorological incidents.

The knowledge base of the rule-based system contains:

- Road network. Defines and identifies:

* The segments and links of the road network associated with each RS. For each segment there are different attributes as number of lanes, road width, verges, speed limits, etc.

* Alternative routes which allow redirecting traffic from one point to another in the road network.

* Singular points as, rest areas where you can store vehicles in the event of an extended cut of the road network, steps from the middle where you can redirect traffic direction or control points.

- Local systems. All the local systems that compound the complete system and the equipment of all of them are defined here.

- Action Protocols. Different incidents (rain, snow, wind, etc.) controlled by the TCC and the action protocols for each incident are defined here.

The fact base of the rule-based system contains the alerts sent by any of its RS.

In case the Manager receives an advice from one of its RS, it has to check if the problem is extended to areas controlled by other of its RS. In that case, it has to extend the affected area and warn the users of the road network about the scope of the meteorological problem. 
Once the Manager agent knows the affected area it has to decide the measures to avoid incidents and send a message to the affected local systems indicating those measures.

In case there is a breakdown in the communications with all local systems, the Manager would not receive any information, but the system will still aware the users about weather problems, due to the capability of decision of the local systems.

\subsection{Interface agent}

The Interface agent is used to show the operators the result of the execution of the other agents.

The Interface agent communicates with an application that shows using Google Maps [10] the current traffic situation. That is: road network, traffic information, incidents, meteorological alerts, VMS and the messages shown in each VMS.

When the Manager agent takes a decision about the measures to take, it sends a message to the Interface agent. This agent will receive the message and show it in a Google Maps application, so the road operator can see the effects and the action protocol evolution.

\subsection{DF agent}

The directory facilitator (DF) provides a yellow pages service by means if which an agent can register, deregister and search for other agents or services in a MAS environment. In the implementation of this system it has been used the DF JADE agent specified by FIPA [21].

\section{Implementation}

The system has been implemented using a JADE [23] platform. JADE is a software framework to develop agent applications in compliance with FIPA specifications for interoperable intelligent multiagent systems.

The inference engine for the rule-based system has been implemented using Drools [22]. Drools is a JBoss Rules engine that facilitates the implementation and modification of the rules by non-experts. Drools has been chosen because it is a Java application, so it is easy to combine with the JADE platform. Besides, Drools allows the creation of an interface, so the experts in traffic can introduce the traffic rules themselves. This way, when there are any changes on the rules, there is no need for a programmer to introduce the new rules on the system.

The system's interface uses web application that displays the MAS and the traffic status via Google Maps. To this end, the application consists of a servlet that processes web requests, updating the values of the equipment every 10 seconds, and various JavaServer Pages that allows the access to the private data of the servlet and shows them at a map in Google Maps.

This way, when the operator starts the application, he sees a Google Maps centered in the road network he is controlling. This map has the normal controls of a Google Maps plus a specific menu to show all the equipment of the road. So the operator can choose if he wants to see the VMS, the RS, the meteorological stations or all them at the same time. Clicking on the equipment, an information bubble is opened showing all the values of the element in that specific moment.

For example, Fig. 3 presents an example of the graphic interface. In this figure two VMS and two MS of the road network A-3 are showed. Clicking in the equipment, a balloon is opened containing the information of that element. From the information of the balloon, we know that VMS is located in the $\mathrm{km} 213$ of the A-3 road network and, currently, it is showing an alert about a strong rain in $15 \mathrm{~km}$. In the left of the graphic interface, there is a special area showing all the incidences of the area of the map are shown.

\section{A Study case: The Spanish A-3 freeway}

In order to evaluate the proposed MAS, a real freeway has been modeled. The Spanish A-3 motorway has been chosen because it presents the desired characteristics: two lanes per sense, several segments including intersections and junctions, different equipment and the possibility of use alternative itineraries. The model developed includes:

- 2 main roads A-3 and A-33.

-16 Segments.

- 20 Links.

- 13 Meteorological stations.

- 13 Remote stations

-22 VMS.

- Real TMP for weather problems in the A-3 freeway.

The modeled network and their characteristics are adequate to test the system because it has 13 local systems distributed across the network, connected to one TCC. 
$\underline{8}$ L. Mart et al. / A multi-agent system for managing adverse weather situations on the road network

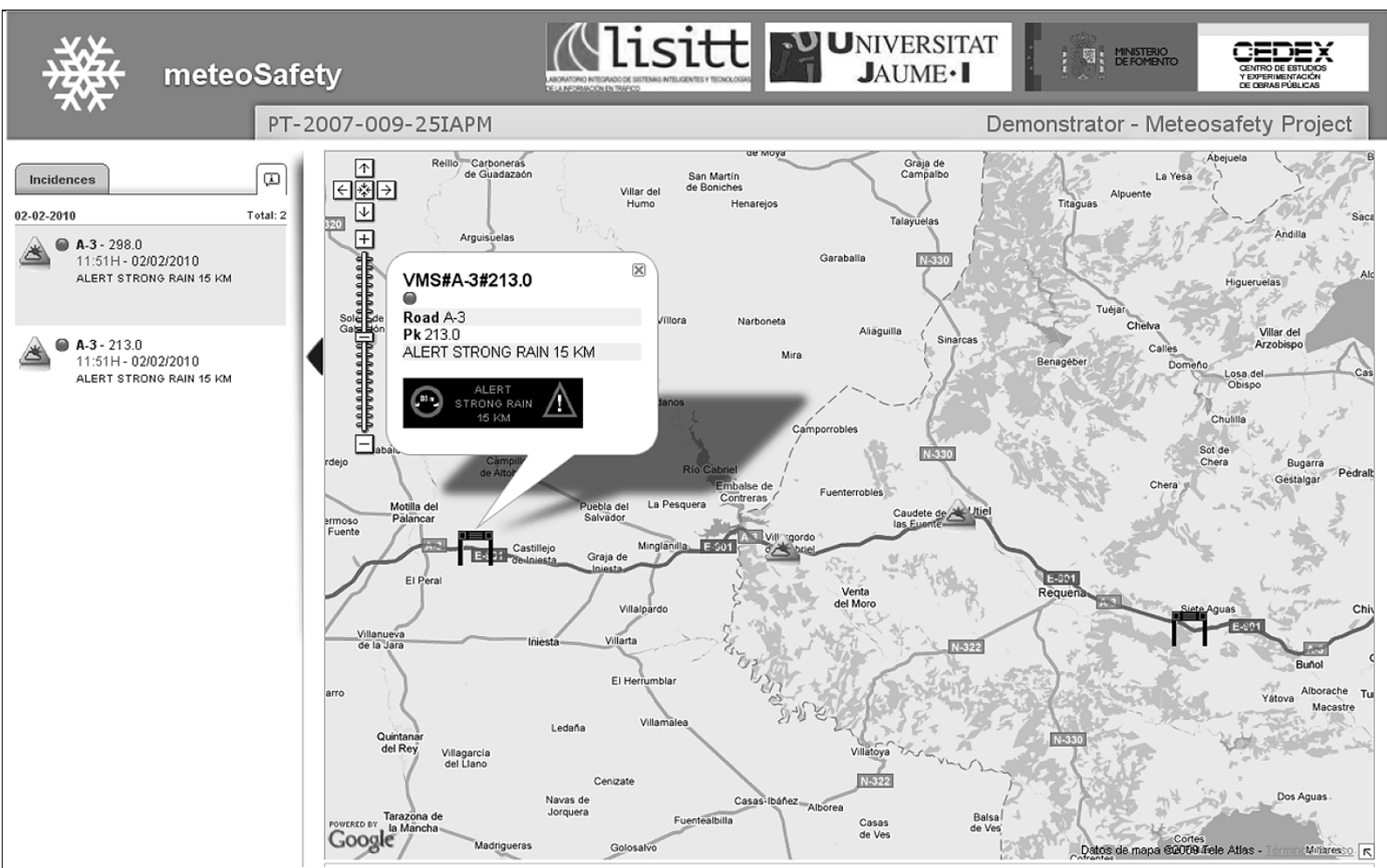

Fig. 3. Example of the graphic interface. A segment of the Spanish A-3 road network from Madrid to Valencia is showed.

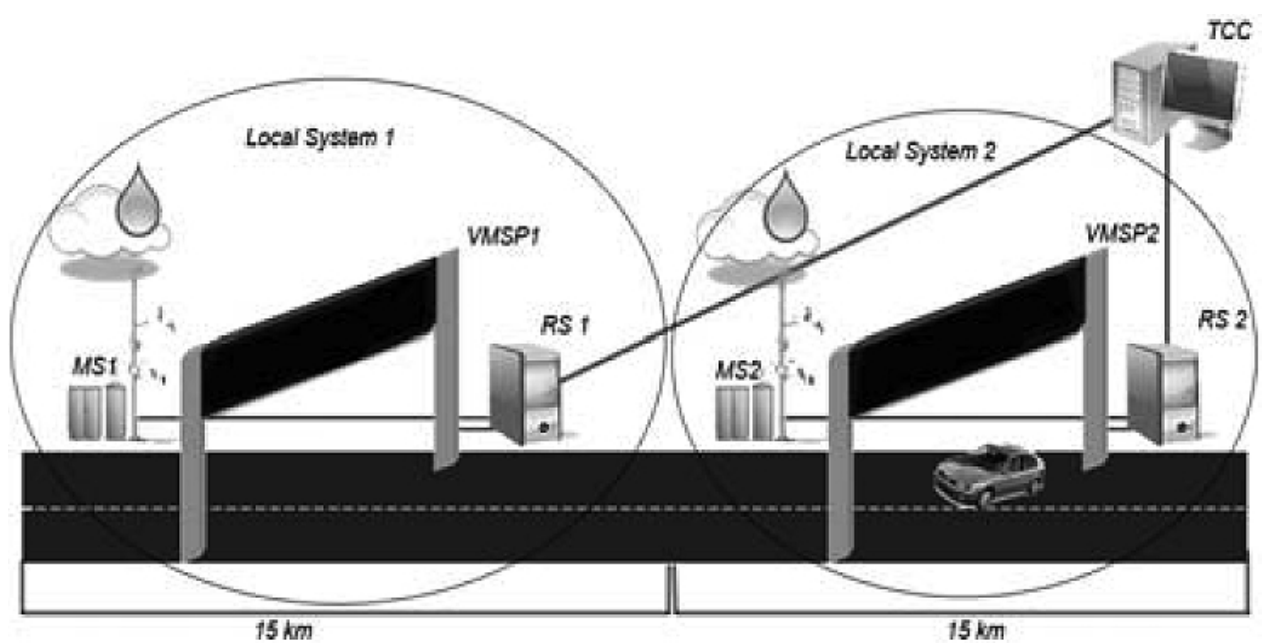

Fig. 4. A part of the road network used to evaluate the MAS prototype. In this case, there are two local systems with one VMS and one MS, connected to the TCC.

controlling two main roads. The system has been tested in several situations: a) in a coordinated way, where all local systems and the TCSs provide a generic solution, b) one local system works autonomously and the rest works coordinately; and c) all local systems work autonomously.
In order to run the simulations, data provided by meteorological stations have been reproduced using real files. Furthermore, specific weather incidences were simulates to test new adverse conditions

Figure 4 shows a part of the road network used to evaluate the MAS prototype. In this case, there are two 


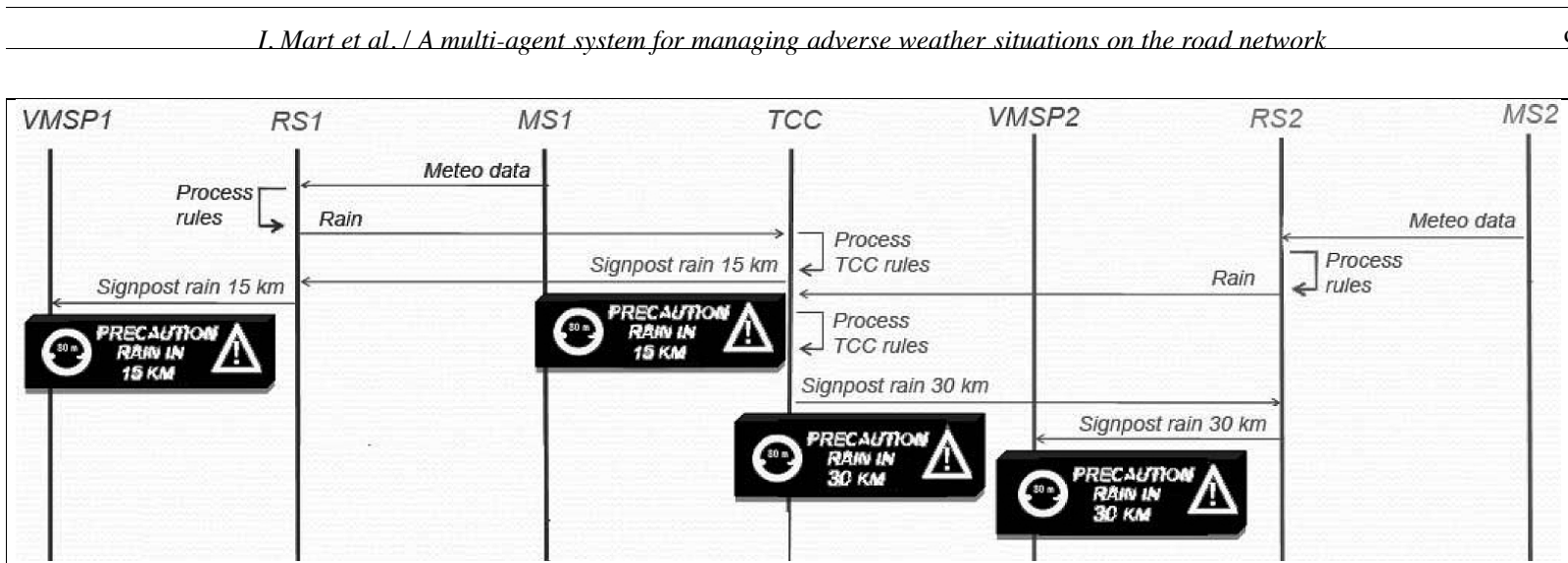

Fig. 5. Exchange of messages between the elements of the MAS in the study case of Fig. 3.

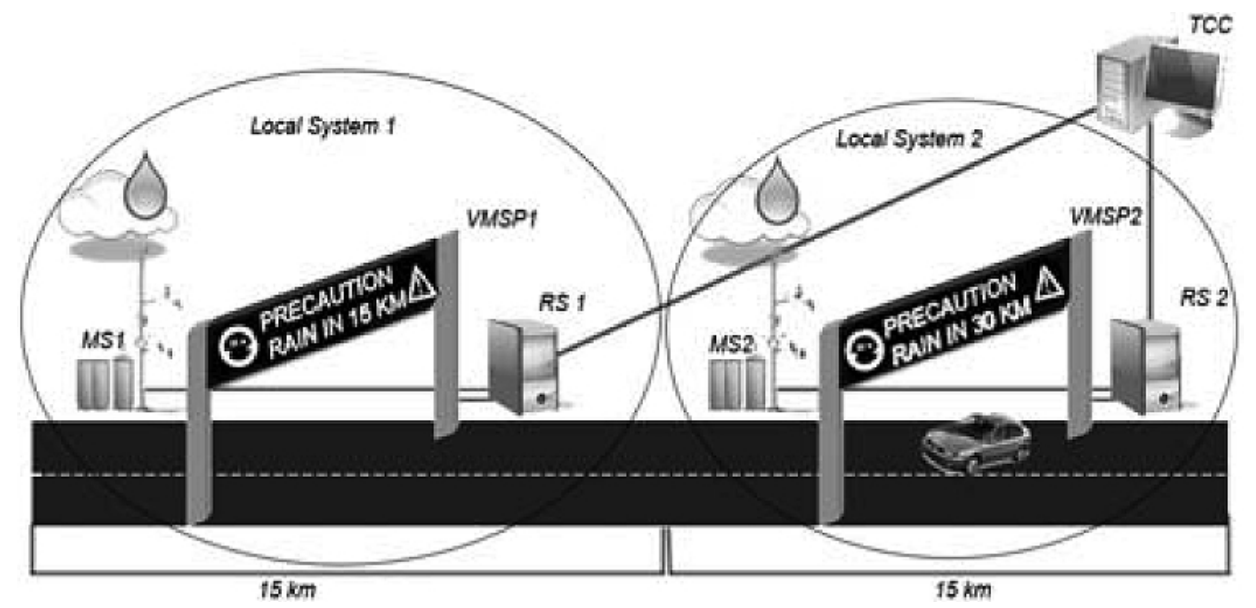

Fig. 6. State of the VMPS of the road network after the analysis by the TCC.

local systems, each one with one VMS and one MS, both connected to the TCC. Traffic flow is from right to left, so vehicles drive from the local system 2 to the local system 1.

In this scenario, a rain alert is detected by both local systems and both RS send the alert to the TCC. When the TCC receives the rain alerts it realizes that the rain is a problem in both local systems, so the area affected by the rain covers $30 \mathrm{~km} ; 15 \mathrm{~km}$ covered by the first local system and $15 \mathrm{~km}$ covered by the second one.

The TCC decides to show a message alerting about the $30 \mathrm{~km}$ of rain in the VMS2, so the users of the road network will know the scope of the problem. Once the users arrive to the local system 1 , there are only $15 \mathrm{~km}$ more of rain, so the VMS1 has to alert about rain in $15 \mathrm{~km}$.

Figure 5 presents the message exchange between all the elements of the system. MS1 sends the data of its sensors to the RS1. Once RS analyzes the data, it sets a meteorological alert about rain, and sends it to the TCC. Few seconds later, the MS2 sends its weather data to RS2, which realizes that it's raining, and sends a rain alert to TCC. The TCC analyzes the alert sent by RS1 and order to signpost in VMS1 "Warning rain in $15 \mathrm{~km}$ ". When the TCC analyzes the alert sent by the $\mathrm{RS} 2$, it realizes that it is raining in the $30 \mathrm{~km}$ covered by both local systems, so it orders to signpost in VMS2 "Warning, rain in $30 \mathrm{~km}$ ".

Figure 6 shows the result of the execution of the taken actions to avoid road incidents because of the rain. In case that a human operator had taken the actions to warn the users about the rain by his own, the result would have been the same. This proves that the MAS prototype is working correctly.

\section{Conclusions and future work}

In this paper it has been exposed the need of developing autonomous intelligent decision support systems 
10 L. Mart et al. I A multi-agent system for managing adverse weather situations on the road network

for traffic management and control. Real situations require local autonomous systems, due to the possibility of a breakdown in the communications between the road equipment and the TCC.

There are several characteristics of the proposed MAS system that improve other ATMS reviewed on this paper.

First, the proposed system is designed as a set of local systems, distributed among the road network. Each local system is composed of different equipment. Basically, it has a RS agent that uses the VMS and MS agents to monitor and manage its coverage area.

These local systems are connected to the TCC by the RS. When a weather incident appears, the affected local systems work coordinately with the TCC to decide the best actions to solve, or at least to minimize, the incident consequences.

Besides, if there appear problems in the operational system (communication breakdowns, HW failures, SW problems ...), each local system continues working. They have the ability of decide by themselves what alerts show and where to signpost the alerts. This way, users will always be warned about any meteorological issue on the road network.

Second, the rule-based increases the soundness of the proposed autonomous system. These kinds of systems require a high level of data quality (data availability, reliability, accuracy, completeness ... ) in order to take the adequate autonomous decisions. Thus, in order to certify the data quality of the alerts, a new control procedure, on the weather data, has been performed.

This control is carried out by combining two different types of rules: correlation and coherence rules. Correlation rules are focused on the analysis of the raw weather data related to historic values obtained by the same MS. Coherence rules are focused on finding correspondences between different weather sensors of the MS.

Combining the result of the rules and a risk factor, a matrix is obtained to establish a priority level for each alert. This way, every local system is able to classify several alerts, in order to have a priority list to act on them.

Furthermore, the inference engine used for the rulebased system is Drools. Drools allows the creation of a friendly specific interface to manage the rules. This way, road operators are able to introduce or modify rules without the necessity to reprogramming the rulebased system every time that a change in the rules has to be done.

The evaluation of the proposed MAS has been done by comparing the real actions of the human road op- erator dealing with a meteorological incident with respect to the actions proposed by the MAS. Concretely, messages selected by a local system to be displayed in each VMS where analyzed and as a consequence, some minor modifications where done in the RS agent rules.

Moreover, the proposed MAS has been analyzed acting coordinately. The MAS is able to manage the complete signalization of the modeled road network in a fast and coordinate way, overcoming the way a human road operator usually performs the same actions.

However, when an alert certainty has medium values and a medium or low risk factor, some rules are activated by the MAS that imply that some actions are proposed to be taken. But in the same situation the human road operator wouldn't take any actions. So, a new matrix is being studied in order to define more detailed the uncertainly situations.

The distributed architecture of the MAS makes it scalable. New agents and functionalities could be added in an easy way. Concretely, a new agent is being designed to serve as interface between the MAS and the traffic police patrols. This agent would send the alerts to the police patrols, so road safety will be improved. Moreover, weather data provided by police patrol (i.e. coming from a place without MS) would be also send to this agent to be integrated with the rest of the data available in the MAS.

The proposed MAS manages traffic incidents due to weather problems inside the coverage road network of the TCC. However, the temporal evolution of the incident may cover more than a TCC area. That should imply the execution of coordinated traffic management strategies between several TCCs. These coordinated strategies are being studied to be managed in an automated and autonomous way. It is necessary not only define the agreements about the traffic management plan to be activated in each situation, but also the way to reach this agreement between the different systems elements.

The VMS agent rules are also being improved. In the MAS exposed in this paper, regulation messages are only fired due to weather situations. However, there exist intrinsic relations between levels of service, weather parameters and basic traffic parameters (density, flow, speed) $[6,15,24]$. These relations are being analyzed in order to add new VMS rules to deal with them.

\section{Acknowledgement}

This research has been supported by the CEDEX project METEOSAFETY: estudio para la reducción de 
accidentes frente a condiciones meteorológicas adversas (METEOSAFETY: study to reduce accidents in case of weather problems) with reference PT-2007-00925IAPM

\section{References}

[1] H. Adeli and A. Karim, Fuzzy-Wavelet RBFNN Model for Freeway Incident Detection, Journal of Transportation Engineering, ASCE 126(6) (2000), 464-471.

[2] H. Adeli and A. Samant, An Adaptive Conjugate Gradient Neural Network - Wavelet Model for Traffic Incident Detection, Computer-Aided Civil and Infrastructure Engineering 15(4) (2000), 251-260.

[3] H. Adeli and X. Jiang, Neuro-Fuzzy Logic Model for Freeway Work Zone Capacity Estimation, Journal of Transportation Engineering, ASCE 129(5) (2003), 484-493.

[4] E. Belda and V.R. Tomás, Using Intelligent Transport Systems to improve road safety, in: Road Safety and Simulation RSS07 (2007).

[5] E. Cascetta and A. Papola, A Trip Distribution Model Involving Spatial and Dominance Attributes, Computer-Aided Civil and Infrastructure Engineering 23(2) (2008), 116-124.

[6] FHWA. Road Weather Management Overview http://ops. fhwa.dot.gov/Weather/overview.htm.

[7] L.A. García and F. Toledo, Space-Temporal Urban Traffic Regulation Problem Identification using Qualitative Data and Temporal Intervals. In International Conference on Artificial Intelligence-2000. USA, 2000.

[8] A. García and D. Teruel, FIPA-compliant MAS development for road traffic management with a knowledge-based approach: TRACK-R Agents for traffic information. In "Open Agent Systems'03" AgentCities workshop at AAMAS-03, 2003.

[9] J.C. Giarratano and G. Riley, Expert Systems, Principles and Programming, 2005.

[10] Google Maps API. http://code.google.com/intl/es-ES/apis/ maps/.

[11] J. Hernández, J. Cuena and M. Molina, Real-time traffic management through knowledge-based models: the TRYS approach. In Tutorial on Intelligent Traffic Management Models, the 11th Mini-Euro Conference on Artificial Intelligence in Transportation Systems and Science, 1999.

[12] X. Jiang and H. Adeli, Freeway Work Zone Traffic Delay and Cost Optimization Model, Journal of Transportation Engineering, ASCE 129(3) (2003), 230-241.

[13] X. Jiang and H. Adeli, Object-Oriented Model for Freeway Work Zone Capacity and Queue Delay Estimation, ComputerAided Civil and Infrastructure Engineering 19(2) (2004), 144 156.
[14] A. Kesting and M. Treibe, How Reaction Time, Update Time. and Adaptation Time Influence the Stability of Traffic Flow. Computer-Aided Civil and Infrastructure Engineering 23(2) (2008), 125-137.

[15] M. Kyte, Z. Khatib, P. Shannon and F. Kithener, Effect of Weather on Free-Flow Speed. Transportation Research Record 1776, Transportation Research Board (2001), 60-68.

[16] H.X. Liu and A. Danczyk. Optimal Sensor Locations for Freeway Bottleneck Identification, Computer-Aided Civil and Infrastructure Engineering 24(8) (2009), 535-550.

[17] I. Martí, V.R. Tomás, L.A. García and J.J. Martínez, A Rulebased Multi-agent System for Local Traffic Management. LNCS on Intelligent Data Engineering and Automated Learning (2009), 502-511.

[18] B. McQueen, Intelligent transportation systems architecture In Artech House Books (1999).

[19] Q. Meng and X. Wang, Sensitivity Analysis of Logit-based Stochastic User Equilibrium Network Flows with Entry-exit Toll Schemes, Computer-Aided Civil and Infrastructure Engineering 23(2) (2008), 138-256.

[20] S. Moreno, F. Toledo, F. Rosisch and G. Martín, Qualitative simulation for Temporal Reasoning in Urban Traffic Control. in: Qualitative Reasoning and Decision technologies, N.Piera Carreté and M.G. Singh, eds, CIMNE, Barcelona, (1993).

[21] P.D. O'Brien and R. Nicol, FIPA - Towards a Standard for Software Agents, BT Technology Journal 16 (1998).

[22] M. Proctor, Relational Declarative Programming with JBos\$ Drools. In SYNASC '07: Proceedings of the Ninth International Symposium on Symbolic and Numeric Algorithms for Scientific Computing. Timisoara, Romania, (2007).

[23] M. Rinard and M. Lam, The design, implementation, and evaluation of Jade. In ACM Transactions on Programming Languages and Systems 20(3) (1998), 483-545.

[24] H. Rakha, M. Farzaneh, M. Arafeh and E. Sterzin, Inclement Weather Impacts on Freeway Traffic Stream Behavior. TRB 2008 Annual Meeting, (2008).

[25] S. Ritchie, A knowledge-based decision support architecture for advanced traffic management, In Transportation research 24(1) (1990), 27-37.

[26] A. Samant and H. Adeli, Feature Extraction for Traffic Incident Detection using Wavelet Transform and Linear Discriminant Analysis, Computer-Aided Civil and Infrastructure Engineering 15(4) (2000), 241-250.

[27] V.R. Tomás and L.A. García, A cooperative multiagent system for traffic management and control. In AAMAS '05: Proceedings of the fourth international joint conference on Autonomous agents and multiagent systems, Netherlands (2005). $52-59$.

[28] M. Wachs, J.M. Samuels and R.E. Skinner, Highway Capacity Manual 2000. Transportation Research Board, 2000. 